PROCEEDINGS OF THE

AMERICAN MATHEMATICAL SOCIETY

Volume 126, Number 7, July 1998, Pages 2039-2046

S 0002-9939(98)04403-7

\title{
SOME NEARLY BOOLEAN ORTHOMODULAR POSETS
}

\author{
PAVEL PTÁK
}

(Communicated by Palle E. T. Jorgensen)

\begin{abstract}
Let $L$ be an orthomodular partially ordered set ("a quantum logic"). Let us say that $L$ is nearly Boolean if $L$ is set-representable and if every state on $L$ is subadditive. We first discuss conditions under which a nearly Boolean OMP must be Boolean. Then we show that in general a nearly Boolean OMP does not have to be Boolean. Moreover, we prove that an arbitrary Boolean algebra may serve as the centre of a (non-Boolean) nearly Boolean OMP.
\end{abstract}

\section{INTRODUCTION AND BASIC NOTIONS}

Orthomodular posets (OMPs) are certain "nondistributive" generalizations of Boolean algebras. They are sometimes called "quantum logics" when viewed as event structures of quantum experiments. The states of the experiments are then associated with probability measures on the corresponding OMPs, called "states" for this reason, which do not in general enjoy standard Boolean features. For instance, they do not have to be subadditive (a state $s$ on an orthomodular poset $L$ is said to be subadditive if for any $a, b \in L$ there exists $c \in L$ such that $c \geq a, c \geq b$ and $s(c) \leq s(a)+s(b))$. In fact, the subadditivity of all states is quite a strong condition imposed on $L$. If, moreover, we require $L$ to be set-representable, which is a conceptually important "Boolean-like" condition, we find $L$ to be intrinsically very near a Boolean algebra. A natural question arises whether these "nearly Boolean" OMPs do not have to be Boolean. This turned out to be a fairly nontrivial problem. Moreover, since subadditive states can be viewed as "strongly Jauch-Piron states" (see [1] and [13] for comments on the Jauch-Piron property in quantum theories), the problem has a bearing on quantum axiomatics. We show in this paper that non-Boolean nearly Boolean OMPs exist abundantly. (Observe that the $\sigma$-complete version of this problem, which is quite different in its mathematical nature, has been solved in [3]. Notice also that for lattices the abundance of subadditive states implies distributivity [14], and therefore in this case one finds oneself in the realm of Boolean algebras.)

It should be noted that the construction we present here is not entirely new the technique of [6] and [7] designed for Jauch-Piron states is used as an essential

Received by the editors December 16, 1996.

1991 Mathematics Subject Classification. Primary 28A60, 06C15, 81P10.

Key words and phrases. Orthomodular partially ordered set, Boolean algebra, state (= finitely additive probability measure), subadditivity.

The author acknowledges the support by the grant GA 201/96/0117 of the Czech Grant Agency.

(C)1998 American Mathematical Society 
tool. We have adapted it to our purpose in some places. Explicit novelties seem to be Th. 2.1 (v), Prop. 2.2 and Th. 2.5.

Let us now introduce the notions we shall need in the sequel.

1.1. Definition. Let $\Omega$ be a set and let $\Delta$ be a collection of subsets of $\Omega$. The pair $(\Omega, \Delta)$ is called a set-representable orthomodular poset (a SROMP) if the following three conditions are satisfied:

(i) $\Omega \in \Delta$,

(ii) if $A \in \Delta$, then $\Omega-A \in \Delta$,

(iii) if $A, B \in \Delta$ and $A \cap B=\emptyset$, then $A \cup B \in \Delta$.

The SROMPs have proved to be of a considerable conceptual value for both noncommutative measure theory and quantum axiomatics (see [11] and [4]). It can be easily shown ([13]) that the SROMPs are exactly those (general) orthomodular posets which admit a set-theoretic representation with set-theoretic union and settheoretic orthocomplementation for the OMP operations. Obviously, a SROMP is a Boolean algebra exactly when it is closed under the formation of intersections.

1.2. Definition. Let $(\Omega, \Delta)$ be a SROMP. By a state on $(\Omega, \Delta)$ we mean a mapping $s: \Delta \rightarrow\langle 0,1\rangle$ such that

(i) $s(\Omega)=1$,

(ii) $s(A \cup B)=s(A)+s(B)$ provided $A, B \in \Delta$ and $A \cap B=\emptyset$.

Let us denote by $\mathcal{S}(\Omega, \Delta)$ the set of all states on $(\Omega, \Delta)$. We will often write simply $\mathcal{S}(\Delta)$ instead of $\mathcal{S}(\Omega, \Delta)$ if $\Omega$ is clear from the context or not needed in the reasoning. By a standard argument, $\mathcal{S}(\Delta)$ is easily seen to be a convex set compact in the pointwise topology. We will use this fact a few times in the sequel.

1.3. Definition. Let $(\Omega, \Delta)$ be a SROMP and let $s \in \mathcal{S}(\Delta)$. We say that the state $s$ is subadditive if the following condition is satisfied: If $A, B \in \Delta$, then there is a set $C \in \Delta$ such that $C \supset A \cup B$ and, moreover, $s(A)+s(B) \geq s(C)$.

If $(\Omega, \Delta)$ is a Boolean algebra, then all states on $\Delta$ are subadditive (we simply take $A \cup B$ for $\mathrm{C}$ ). In SROMPs, we typically have both subadditive and nonsubadditive states. The following example well illustrates the situation.

1.4. Example. Let $r$ be a positive rational number. Let $\Omega=\langle 0, r\rangle \times\langle 0, r\rangle$ and, further, let $\Delta=\{A \subset \Omega \mid A$ is a Lebesgue measurable set in $\Omega$ such that $\mu(A)$ is a rational number, where $\mu$ is the Lebesgue measure $\}$. Then a routine verification gives that $(\Omega, \Delta)$ is a SROMP. Fix a set $B \in \Delta$ with $\mu(B)>0$, and define a mapping $s_{B}: \Delta \rightarrow\langle 0,1\rangle$ by putting $s_{B}(A)=\frac{\mu(B \cap A)}{\mu(B)}$. Then $s_{B}$ is a state on $(\Omega, \Delta)$. It can be shown that $s_{B}$ is subadditive if, and only if, $\mu(B)$ is a rational number.

Let us now introduce the central notion of this paper.

1.5. Definition. Let $(\Omega, \Delta)$ be a SROMP. If every state $s$ on $\Omega, \Delta)$ is subadditive, then $(\Omega, \Delta)$ is called nearly Boolean.

In what follows, we will analyse how "near" the nearly Boolean OMPs are to Boolean algebras. 


\section{Results}

The following theorem lists the hitherto known cases in which nearly Boolean implies Boolean (the statement (vi) is auxiliary). We provide sketches of all proofs for the sake of completeness, though some of the proofs can be extracted from [8] and [12]. (Let us recall that a maximal Boolean subalgebra of an OMP is called a block. Obviously, every Boolean subalgebra of an OMP can be enlarged to a block.)

2.1. Theorem. Let $(\Omega, \Delta)$ be a SROMP. Then the following statements hold true:

(i) If $\Delta$ is a lattice, then $\Delta$ is Boolean.

(ii) If $\Omega$ is countable, then $\Delta$ is Boolean.

(iii) If $\Delta$ posseses only finite blocks, then $\Delta$ is Boolean.

(iv) If $\Delta$ posseses only finitely many blocks, then $\Delta$ is Boolean.

(v) If $\Delta$ is $2^{\chi_{0}}$-complete, then $\Delta$ is Boolean.

(vi) If $A_{1}, A_{2}, \ldots, A_{n}$ are sets from $\Delta$, then there is a finite collection of sets from $\Delta$, call them $B_{1}, B_{2}, \ldots, B_{n}$, such that $\bigcap_{i \leq n} A_{i}=\bigcup_{j \leq m} B_{j}$. If, moreover, a positive $\varepsilon$ is given, then the sets $B_{1}, B_{2}, \ldots, B_{m}$ can be chosen so that for any state $s \in \mathcal{S}(\Delta)$ such that $s\left(A_{i}\right)=1$ for each $i \leq n$ there is a $B_{j}(j \leq m)$ such that $s\left(B_{j}\right)>1-\varepsilon$.

Proof. (i) Take two sets $A, B \in \Delta$ and consider $A \wedge B$. We want to show that $A \wedge B=A \cap B$. Suppose that it is not the case. Then there is a point $p \in \Omega$ such that $p \in(A \cap B)-(A \wedge B)$. Consider the two-valued state $s_{p}$ concentrated at the point $p$. Thus, $s_{p}(c)=1$ if, and only if, $p \in C$. Then $s_{p}(\Omega-A)=s_{p}(\Omega-B)=0$. Since $s_{p}$ is supposed to be subadditive, there is a set $\tilde{C}$ such that $\tilde{C} \supset(\Omega-A) \cup(\Omega-B)$ and $s_{p}(\tilde{C}) \leq s_{p}(\Omega-A)+s_{p}(\Omega-A)=0$. It follows that $s_{p}(\tilde{C})=0$. If we put $C=\Omega-\tilde{C}$, then $s_{p}(C)=1$ and therefore $C \neq \emptyset$. Moreover, $C \subset A \cap B$ and $p \in C$. By the definition of infima, $C \subset A \wedge B$, and this is a contradiction. We have shown that $A \cap B \in \Delta$ for any $A, B \in \Delta$, and this means that $(\Omega, \Delta)$ is Boolean.

(ii) Suppose that $A, B \in \Delta$ and $A \cap B \neq \emptyset$. Put $A \cap B=\left\{p_{n} \mid n \in N\right\}$ and

$$
s=\sum_{n \in N} \frac{1}{2^{n}} s_{p_{n}},
$$

where $s_{p_{n}}$ are states concentrated at $p_{n}$. Obviously, $s(A)=s(B)=1$, and the subadditivity of $s$ guarantees a set $C$ such that $C \in \Delta, C \subset A \cap B$ and $s(C)=1$. But then $C=A \cap B$, and this is what we wanted to show.

For the proof of the remaining properties, let us first verify the condition (vi) of Th. 2.1.

(vi) Let us first observe a simple fact which we formulate as a lemma.

Lemma. Suppose that $(\Omega, \Delta)$ is a SROMP. Suppose that $\left\{A_{1}, A_{2}, \ldots, A_{n}\right\} \subset \Delta$ and suppose further that $s\left(A_{1}\right)=s\left(A_{2}\right)=\cdots=s\left(A_{n}\right)=1$ for a subadditive state $s \in S(\Omega, \Delta)$. Then there is a set $B, B \in \Delta$, such that $B \subset \bigcap_{i \leq n} A_{i}$ and $s(B)=1$.

Proof of the Lemma. We will first prove the lemma for two sets, $A_{1}$ and $A_{2}$. Suppose that $s\left(A_{1}\right)=s\left(A_{2}\right)=1$. Then $s\left(\Omega-A_{1}\right)=s\left(\Omega-A_{2}\right)=0$. Since $s$ is subadditive, there is a set $\tilde{B}$ such that $\tilde{B} \supset\left(\Omega-A_{1}\right) \cup\left(\Omega-A_{2}\right)$ and $s(\tilde{B}) \leq$ $s\left(\Omega-A_{1}\right)+s\left(\Omega-A_{2}\right)=0$. If we put $B_{1}=\Omega-\tilde{B}$, then $B_{1} \subset A_{1} \cap A_{2}$ and $s\left(B_{1}\right)=1$.

Let us now proceed inductively. Suppose that for $A_{1}, A_{2}$ we have the required set $B_{1}$. Let us consider the sets $B_{1}, A_{3}$, and find the set $B_{2}$ such that $B_{2} \subset B_{1} \cap A_{3} \subset$ 
$A_{1} \cap A_{2} \cap A_{3}$ and $s\left(B_{2}\right)=1$. Going on in this way, we finally take $B_{n-1}$ for $B$, which establishes the lemma.

Let us return to the proof of of Th. 2.1(vi). Suppose that $\left\{A_{1}, A_{2}, \ldots, A_{n}\right\} \subset$ $\Delta$. If $\bigcap_{i \leq n} A_{i}=\emptyset$, then there is nothing to be proved. Suppose therefore that $\bigcap_{i \leq n} A_{i} \neq \emptyset$. Put $\mathcal{S}=\left\{s \mid s \in \mathcal{S}(\Omega, \Delta)\right.$ and $\left.s\left(A_{1}\right)=s\left(A_{2}\right)=\cdots=s\left(A_{n}\right)=1\right\}$. Obviously, $\mathcal{S} \neq \emptyset$. Moreover, since $\mathcal{S}$ is a closed subset of the (compact) set $\mathcal{S}(\Omega, \Delta)$, the set $\mathcal{S}$ is also compact. Put $\mathcal{B}=\left\{B \mid B \in \Delta\right.$ and $\left.B \subset \bigcap_{i<n} A_{i}\right\}$ and set, for any $B \in \mathcal{B}, S_{B}=\{s \in \mathcal{S}(\Omega . \Delta) \mid s(B)>0\}$. Every set $S_{B}$ is obviously an open set in $\mathcal{S}$ and, by our lemma, $\mathcal{S}=\bigcup_{B \in \mathcal{B}} S_{B}$. This means that $\left\{S_{B} \mid B \in \mathcal{B}\right\}$ is an open covering of $\mathcal{S}$, and therefore there is a finite collection $B_{1}, B_{2}, \ldots, B_{m}$ such that $\mathcal{S}=\bigcup_{i \leq m} S_{B_{i}}$. It remains to be shown that $\bigcup_{i \leq m} B_{i}=\bigcap_{i \leq n} A_{i}$. If this is not the case, then there is a point $p \in \bigcap_{i<n} A_{i}-\bigcup_{i<m} B_{i}$. Let us consider the state $s_{p}$ concentrated at $p$. It follows that $s_{p}\left(A_{i}\right)=1$ for any $i, i \leq n$, and therefore $s_{p} \in \mathcal{S}$. On the other hand, $s_{p}\left(B_{i}\right)=0$ for any $B_{i}(i \leq m)$, and this is absurd. (For the additional statement of Th. 2.1(vi), we simply take the sets $S_{B}^{\varepsilon}=\{s \in \mathcal{S} \mid s(B)>1-\varepsilon\}$ in the above proof instead of $S_{B}$.)

(iii) Let us suppose that $(\Omega, \Delta)$ possesses only finite blocks. If $A, B \in \Delta$ and $A \cap B \neq \emptyset$, then there is a nonempty set $C_{1} \in \Delta$ such that $C_{1} \subset A \cap B$ (Th. $2.1(\mathrm{vi}))$. If $\left(A-C_{1}\right) \cap\left(B-C_{1}\right)=\emptyset$, we are done, for $A \cap B=\Delta$. If not, then there is a nonempty set $C_{2}$ such that

$$
C_{2} \subset\left(A-C_{1}\right) \cap\left(B-C_{1}\right) .
$$

If $\left(A-\left(C_{1} \cup C_{2}\right)\right) \cap\left(B-\left(C_{1} \cup C_{2}\right)\right)=\emptyset$, we are done. If not, then there is a nonempty set $C_{3}$ such that $C_{3} \subset\left(A-\left(C_{1} \cup C_{2}\right)\right) \cap\left(B-\left(C_{1} \cup C_{2}\right)\right)$, etc. If $\bigcup_{i \leq n} C_{i}=A \cap B$ for some $n \in N$, we are done. If not, then there is a countable collection $\left\{C_{i} \mid i \in N\right\}$ of mutually disjoint sets which belong to $\Delta$. But then the Boolean algebra generated by $\left\{C_{i} \mid i \in N\right\}$ in $\Delta$ is infinite, which is a contradiction. Thus, $(\Omega, \Delta)$ must be Boolean.

(iv) Suppose that $(\Omega, \Delta)$ possesses exactly $n$ maximal Boolean subalgebras. Take any two sets $A_{1}, A_{2} \subset \Delta$, and consider Th. 2.1 (vi) for $\varepsilon=\frac{1}{n}$. We see that there is a collection $\left\{B_{1}, B_{2}, \ldots, B_{m}\right\} \subset \Delta$ such that $\bigcup_{j \leq m} B_{j}=A_{1} \cap A_{2}$ and, moreover, for any state $s \in \mathcal{S}(\Delta)$ with $s\left(A_{1}\right)=s\left(A_{2}\right)=1$ there is $B_{j}$ such that $s\left(B_{j}\right)>1-\frac{1}{n}$. Since every $B_{j}(j \leq m)$ must belong to a maximal Boolean subalgebra of $\Delta$, we may (and shall) assume that $m \leq n$ (otherwise we simply take the unions of those $B_{j}$ which belong to the same maximal Boolean subalgebra of $(\Omega, \Delta))$. We may further assume that $B_{j} \neq A_{1} \cap A_{2}$ for any $j(j \leq m)$. This means that there are points $p_{j} \in \Omega(j \leq m)$ such that, for any $j \leq m, p_{j} \in\left(A_{1} \cap A_{2}\right)-B_{j}$. Put $s=\sum_{j \leq m} \frac{1}{m} s_{p_{j}}$ $\left(s_{p_{j}}\right.$ is the state concentrated at $\left.p_{j}\right)$. Then $s\left(B_{j}\right) \leq 1-\frac{1}{m} \leq 1-\frac{1}{n}$ for any $j \leq m$, and this is a contradiction. It follows that $(\Omega, \Delta)$ is a Boolean algebra.

(v) This part strengthens a result of the paper [6], where the author proves the same fact for complete SROMPs. We have not been able to prove (or disprove) the same statement for $\sigma$-complete SROMPs (this problem has already been raised in $[6])$.

Let $(\Omega, \Delta)$ be a $2^{\aleph_{0}}$-complete SROMP. In other words, let $(\Omega, \Delta)$ be closed under the formation of the unions of the pairwise disjoint collections of elements from $\Delta$ consisting of at most $2^{\aleph_{0}}$ elements. Take arbitrary sets $A, B \in \Delta$ and find the collection $B_{1}, B_{2}, \ldots, B_{m} \in \Delta$ such that $\bigcup_{j \leq m} B_{j}=A \cap B$ (Th. 2.1 (vi)). Put 
$\mathcal{B}_{1}=\left\{B_{j} \mid j \leq m\right\}$. We will show that $\bigcup_{j \leq m} B_{j} \in \Delta$, proving that $A \cap B \in \Delta$. This will mean that $(\Omega, \Delta)$ is Boolean.

Form the coarsest partition, $\mathcal{P}_{1}$, of $A \cap B$ that refines the covering $\mathcal{B}_{1}$ of the set $A \cap B$. Thus, $\mathcal{P}_{1}$ consists of the sets $\bigcap_{j \leq m} \tilde{B}_{j}$, where $\tilde{B}_{j}$ is either $B_{j}$ or $\Omega-B_{j}$. Write $\mathcal{P}_{1}=\left\{C_{k} \mid k \leq r\right\}$. By Th. 2.1 (vi), every $C_{k}$ can be expressed as a union of the type $\bigcup_{p \leq s} B_{p}^{k}$ for some $s \in N$, where $B_{p}^{k} \in \Delta$ for any $p, p \leq s$. Obviously,

$$
\bigcup_{\substack{k \leq r \\ p \leq s}} B_{p}^{k}=A \cap B .
$$

Put $\mathcal{B}_{2}=\left\{B_{p}^{k} \mid k \leq r, p \leq s\right\}$ and denote by $\mathcal{P}_{2}$ the coarsest partition of $A \cap B$ that refines $\mathcal{B}_{2}$. Going on in this way, we obtain a countable collection $\mathcal{B}_{1}, \mathcal{B}_{2}, \ldots$ of coverings of $A \cap B$ with the following property. If $P \in \mathcal{B}_{u}(u \in N)$ and $Q \in \mathcal{B}_{v}$ $(v \in N)$ for $u<v$, then the following implication holds true: If $P \cap Q \neq \emptyset$, then $P \subset Q$. It can be easily seen that the sets of the type $P_{1} \cap P_{2} \cap P_{3} \cap \ldots$, where $P_{i} \in \mathcal{B}_{i}$, belong to $\Delta$ (here even $\sigma$-completeness would suffice) and, moreover, these sets constitute a partition of $A \cap B$ into not more than $2^{\aleph_{0}}$ sets. This means that $A \cap B \in \Delta$, and the proof is complete.

The previous results indicate that if "properly" nearly Boolean OMP exist, they must be rather complicated. It therefore seems probable that the following procedure for constructing non-Boolean nearly Boolean OMP can hardly be simplified. The following result, which generalizes step VI in the proof of Theorem 1 in [6], provides an essential argument.

2.2. Proposition. Let $(\Omega, \Delta)$ be a SROMP, and suppose that for any pair $A, B \in$ $\Delta$ there exist an uncountable set $I$ and a collection $C_{\alpha}, D_{\alpha} \in \Delta(\alpha \in I)$ such that the following two conditions are satisfied:

(i) For any $\alpha \in I, C_{\alpha} \cup D_{\alpha}=A \cap B$.

(ii) If $\alpha \neq \beta$, then $C_{\alpha} \cap C_{\beta}=\emptyset$.

Then $(\Omega, \Delta)$ is nearly Boolean.

Proof. Let $s \in \mathcal{S}(\Delta)$ be a state on $\Delta$. We must show that $s$ is subadditive. Suppose that $A, B \in \Delta$. We have to exhibit a set $D, D \in \Delta$, such that $D \supset A \cup B$ and $s(D) \leq$ $s(A)+s(B)$. Let $\tilde{A}=\Omega-A$ and $\tilde{B}=\Omega-B$. Let $C_{\alpha}, D_{\alpha}(\alpha \in I)$ be the collection ensured by the assumption of Prop. 2.2 taken for $\tilde{A}$ and $\tilde{B}$. It can be seen easily that there is an index $\alpha_{0} \in I$ such that $s\left(C_{\alpha_{0}}\right)=0$. Indeed, if $s\left(C_{\alpha}\right)>0$ for any $\alpha \in I$, then there is an infinite set $J, J \subset I$, such that $s\left(C_{j}\right)>\frac{1}{n}$ for some $n \in N$. Since the collection $C_{\alpha}(\alpha \in I)$ consists of mutually disjoint sets, we reach a contradiction with the additivity of $s$. Consider now the set $C_{\alpha}$. Since $C_{\alpha_{0}} \cup D_{\alpha_{0}}=\tilde{A} \cap \tilde{B}$, we see that $\left(\tilde{A}-C_{\alpha_{0}}\right) \cap\left(\tilde{B}-D_{\alpha_{0}}\right)=\emptyset$. It follows that $s\left(\tilde{A}-C_{\alpha_{0}}\right)+s\left(\tilde{B}-D_{\alpha_{0}}\right) \leq 1$ and therefore $s(\tilde{A})-s\left(C_{\alpha_{0}}\right)+s(\tilde{B})-s\left(D_{\alpha_{0}}\right) \leq 1$. Since $s\left(C_{\alpha_{0}}\right)=0$, we obtain $s(\tilde{A})+s(\tilde{B}) \leq 1+s\left(D_{\alpha_{0}}\right)$. Put $D=\Omega-D_{\alpha_{0}}$. Then $D \supset A \cup B$, because $D_{\alpha} \subset \tilde{A} \cap \tilde{B}$. Also, $D_{\alpha}=\Omega-D$ and $\tilde{A}=\Omega-A, \tilde{B}=\Omega-B$. Substituting into the inequality $s(\tilde{A})+s(\tilde{B}) \leq 1+s\left(D_{\alpha}\right)$, we have $s(\Omega-A)+s(\Omega-B) \leq 1+s(\Omega-D)$. This yields $1-s(A)+1-s(B) \leq 1+1-s(D)$, which gives the inequality $s(D) \geq s(A)+s(B)$. The proof of Prop 2.2 is complete.

Before we take up the construction of the desired example, let us recall the following simple but useful fact (see [7] for the definition and other considerations). 
2.3. Proposition. Let $(\Omega, \Sigma)$ be a Boolean algebra of subsets of $\Omega$. Let $G$ be a group. Let $m: \Sigma \rightarrow G$ be a G-valued measure (i.e., $m(\phi)=0$, and $m(A \cup B)=$ $m(A)+m(B)$ whenever $A \cap B=\emptyset)$. Then if $H$ is a subgroup of $G$ such that $m(\Omega) \in H$, then the set $\Delta=\{A \in \Sigma \mid m(S) \in H\}$ is a SROMP.

Proof. Obviously, $\phi \in \Delta$. If $A \in \Delta$, then $m(\Omega-A)=m(\Omega)-m(A) \in H$. Thus, $\Omega-A \in \Delta$. Finally, if $A, B \in \Delta$ and $A \cap B=\emptyset$, then $m(A \cup B)=m(A)+m(B) \in H$. This means that $A \cup B \in \Delta$. The proof is complete.

We are ready to present the example of a non-Boolean nearly Boolean OMP. We omit some technical details, assuming the reader to the familiar with the standard construction of Boolean field product and basic extension technique for measures (see e.g. [5] and [15]).

2.4. Theorem. There is a non-Boolean nearly Boolean OMP.

Proof. Let $P, Q$ be two disjoint uncountable sets of equal cardinality. Put $S=P \cup Q$ and consider the Boolean algebra $\tilde{B}=(S, \tilde{\Sigma})$ where $\tilde{\Sigma}$ consists of finite-cofinite subsets of $S$. Let $Z$ denote the group of whole numbers, and let $\tilde{m}_{1}$ (resp. $\tilde{m}_{2}$ ) be the $Z$-valued measure $\tilde{m}_{1}: \tilde{\Sigma} \rightarrow Z$ (resp., $\tilde{m}_{2}: \tilde{\Sigma} \rightarrow Z$ ) which is determined as follows: $\tilde{m}_{1}(\{x\})=1$ if $x \in P, \tilde{m}_{1}(\{x\})=0$ if $x \in Q$ and $\tilde{m}_{1}(S)=1$ (resp., $\tilde{m}_{2}(\{x\})=0$ if $x \in P, \tilde{m}(\{x\})=1$ if $x \in Q$ and $\left.\tilde{m}_{2}(S)=1\right)$. Now, take countably many copies of the Boolean algebra $\tilde{B}$ and form the corresponding countable Boolean field product $B$. Thus, $B=(T, \Sigma)$, where $T$ denotes the cartesian product of countably many copies of the set $S$ and $\Sigma$ consists of finite disjoint unions of "generalized rectangles" in $T$ (by a generalized rectangle in $T$ we mean a set of the type

$$
A_{1} \times A_{2} \times \cdots \times A_{n} \times \prod_{j \geq n+1} S_{j},
$$

where $A_{j} \in \tilde{\Sigma}$ for $j \leq n$ and $S_{j}=S$ for $j \geq n+1$ ). Since $\tilde{m}_{1}(S)=1$ (resp., $\tilde{m}_{2}(S)=1$ ), we can extend $\tilde{m}_{1}$ (resp., $\tilde{m}_{2}$ ) over the entire $\Sigma$, obtaining measures $m_{1}$ and $m_{2}$ on $\Sigma$. (Indeed, if $V=A_{1} \times A_{2} \times \cdots \times A_{n} \times \prod_{j \geq n+1} S_{i}$ is a generalized rectangle, we put

$$
m_{1}(V)=\prod_{i \leq n} \tilde{m}_{1}\left(A_{i}\right), \quad m_{2}(V)=\prod_{i \leq n} \tilde{m}_{2}\left(A_{i}\right)
$$

and continue in the standard way - see e. g. [15].) Let $m=m_{1}+m_{2}$. Obviously, $m$ is a measure, $m: \Sigma \rightarrow Z$, and $m(T)=2$. Let us denote by $Z_{2}$ the group of even numbers considered as a subgroup of $Z$. Let $\Delta=\left\{A \in Z \mid m(A) \in Z_{2}\right\}$. Then $(T, \Delta)$ is a SROMP (Prop. 2.3). We claim that $(T, \Delta)$ is a non-Boolean nearly Boolean OMP.

First, let us show that $(T, \Delta)$ is not Boolean. Pick a point $x \in P$ and two distinct points $y_{1}, y_{2} \in Q$. Let $V_{1}=\left\{x, y_{1}\right\} \times \prod_{j \geq 2} S_{j}, V_{2}=\left\{x, y_{2}\right\} \times \prod_{j \geq 2} S_{j}\left(S_{j}=S\right.$ for $j \geq 2)$. Then $m\left(V_{1}\right)=m\left(V_{2}\right)=2$, and therefore $A, B \in \Delta$. On the other hand, $m\left(V_{1} \cap V_{2}\right)=m\left(\{x\} \times \prod_{j \geq 2} S_{j}\right)=1$, and therefore $V_{1} \cap V_{2} \notin \Delta$. Thus, $(T, \Delta)$ is not Boolean.

Let us prove that $(T, \Delta)$ is nearly Boolean. We will verify that $(T, \Delta)$ satisfies the assumption of Prop. 2.2. Suppose that $A, B \in \Delta$. If $A \cap B \in \Delta$, then we put $C_{\alpha}=\emptyset, D_{\alpha}=A \cap B$, the index $\alpha$ formally varying over an uncountable set. Suppose that $A \cap B \notin \Delta$. Then $m(A \cap B)$ is an odd number. We have defined $m=m_{1}+m_{2}$, and we also know that $A \cap B$ can be expressed as a disjoint union 
of rectangles, $A \cap B=\bigcup_{j \leq n} R_{j}$. Since there is a rectangle, $R_{j_{0}}$, for which either $m_{1}\left(R_{j_{0}}\right) \neq 0$ or $m_{2}\left(R_{j_{0}}\right) \neq 0$, we see that we can find points $p_{1}, p_{2}, \ldots, p_{m}$ such that the set $V=\left\{p_{1}\right\} \times\left\{p_{2}\right\} \times \cdots \times\left\{p_{m}\right\} \times \prod_{j \geq m+1} S_{j}\left(S_{j}=S\right.$ for $\left.j \geq m+1\right)$ is a subset of $A \cap B$ and, moreover, either $m_{1}(V)=1$ or $m_{2}(V)=1$. Observe that if $m_{1}(V)=1$ then $m_{2}(V)=0$, and if $m_{2}(V)=1$ then $m_{1}(V)=0$.

Suppose that $m_{1}(V)=1$ and $m_{2}(V)=0$ (the other case can be argued similarly). Consider the set $S_{m+1}\left(S_{m+1}=S\right)$ in the above expression of the set $V$. Take the partition $\mathcal{P}, \mathcal{P}=\left\{E_{\alpha} \mid \alpha \in I\right\}$, of $S_{m+1}$ such that the index set $I$ is the set of quadruples of elements of $S_{m+1}$ and the sets $E_{\alpha}$ are defined as follows: Every $E_{\alpha}$ consists of four points, $E_{\alpha}=\left\{x_{1}, x_{2}, x_{3}, x_{4}\right\}$, where $x_{1}, x_{2} \in P$ and $x_{3}, x_{4} \in Q$. For any $E_{\alpha}=\left\{x_{1}, x_{2}, x_{3}, x_{4}\right\}$, let us set

$$
C_{\alpha}=\left\{p_{1}\right\} \times\left\{p_{2}\right\} \times \cdots \times\left\{p_{m}\right\} \times E_{\alpha} \times \prod_{j \geq n+2} S_{j}
$$

and

$$
D_{\alpha}=A \cap B-\left(\left\{p_{1}\right\} \times\left\{p_{2}\right\} \times \cdots \times\left\{p_{m}\right\} \times\left\{x_{1}\right\} \times \prod_{j \geq n+2} S_{j}\right) .
$$

Obviously, $C_{\alpha} \cup D_{\alpha}=A \cap B$ for any $\alpha \in I$, and all sets $C_{\alpha}$ belong to $\Delta$. Since the number $m\left(D_{\alpha}\right)$ can be expressed as a difference of two odd numbers, we see that $m\left(D_{\alpha}\right)$ is an even number and therefore $D_{\alpha} \in \Delta$ for any $\alpha \in I$. The proof is complete.

The above result can be improved by ensuring an arbitrary degree of "absolute compatibility" in nearly Boolean OMPs. In other words, it can be proved that there are nearly Boolean OMPs with arbitrary centres (for the notion of compatibility and centre in OMP, see e.g. [13]). This might be relevant to the logico-algebraic foundations of quantum theories. Also, it establishes a proper class of nearly Boolean OMPs. To keep the exposition short, we assume that the reader is aquainted with the construction of a Boolean power of OMPs (see e.g. [9] and [2]).

2.5. Proposition. Let $L=(\Omega, \Delta)$ be a nearly Boolean OMP. Let B be a Boolean algebra. Then the Boolean power, $P$, of $B$ and $L$ is a nearly Boolean OMP. Moreover, if $C(\Delta)$ denotes the centre of $\Delta$ and if $C(\Delta)=\{0,1\}$, then $C(P)=B$.

Proof. This immediately follows from the description of the states on $P$ (see e.g. [10]) and from compatibility properties of a Boolean power (see e.g. [9] and [2]).

2.6. Theorem. Suppose that $B$ is a Boolean algebra. Then there is a non-Boolean nearly Boolean $O M P, L$, such that $C(L)=B$.

Proof. Observe that the nearly Boolean OMP constructed in Th. 2.3 has a centre consisting only of $\{0,1\}$. The rest follows from Prop. 2.4.

Let us conclude this paper by formulating an open question that arises naturally from Th. 2.3. Can every SROMP be faithfully embedded in a nearly Boolean OMP? (An OMP embedding $e: L \rightarrow K$ is called faithful if $a$ is compatible with $b$ in $L$ exactly when $e(a)$ is compatible with $e(b)$ in $K$.) A positive answer to this question could have a bearing on quantum axiomatics. It should be noted that the $\sigma$-additive version of this question has been positively answered in [3]. 


\section{REFERENCES}

[1] Beltrametti, E., Cassinelli, G., The logic of quantum mechanics, Addison-Wesley, Reading, Massachusetts, 1981. MR 83d:81008

[2] Bruns, G., Greechie, R.J., Harding, J., Roddy, M., Completions of orthomodular lattices, Order 7 (1990), 67-76. MR 92b:06029

[3] DeLucia, P., Pták, P., Quantum probability spaces that are nearly classical, Bull. Polish Acad. Sciences - Math. 40 (2) (1992), 163-173. MR 97i:81015

[4] Gudder, S., Stochastic Methods in Quantum Mechanics, North-Holland, Amsterdam, 1979. MR 84j: 81003

[5] Halmos, P., Measure Theory, Van Nostrand, New York, 1950. MR 11:504d

[6] Müller, V., Jauch-Piron states on concrete quantum logics, Int. Journ. Theor. Phys. 32 (3) (1993), 433-442. MR 94g:81011

[7] Navara, M., Kernel logics, Czechoslovak Math. J., (to appear).

[8] Navara, M., Pták, P., Almost Boolean orthomodular posets, Jour. Pure Applied Algebra 60 (1989), 105-111. MR 90h:06003

[9] Pták, P., Logics with given centers and state spaces, Proc. Amer. Math. Soc. 88 (1983), 106-109. MR 84f:06016

[10] Pták, P., Summing of Boolean algebras and logics, Demonstratio Math. 19 (1986), 349-357. MR 89b:03104

[11] Pták, P., Jauch-Piron property (everywhere!) in the logico-algebraic foundation of quantum theories, Int. Journ. Theor. Phys. 32 (10) (1993), 1985-1991. MR 94j:81024

[12] Müller, V., Pták, P., Tkadlec, J., Concrete quantum logics with covering properties, Int. Journ. Theor. Phys. 31 (5) (1992), 843-854. MR 93a:81021

[13] Pták, P., Pulmannová, S., Orthomodular Structures as Quantum Logics, Kluwer Academic Publishers, Dordrecht/Boston/London, 1991. MR 94d:81018b

[14] Pták, P., Pulmannová, S., A measure theoretic characterization of Boolean algebras among orthomodular lattices, Comment. Math. Univ. Carolinae 35 (1) (1994), 205-208. MR 95i:06014

[15] Sikorski, R., Boolean Algebras, Springer Verlag, Berlin-Heidelberg-New York, 1969. MR 39:4053

[16] Varadarajan, V., Geometry of Quantum Theory I, Van Nostrand, Princeton, 1968. MR $\mathbf{5 7 : 1 1 3 9 9}$

Czech Technical University, Faculty of Electrical Engineering, Department of Mathematics, 16627 Prague 6, Czech Republic

E-mail address: ptak@math.feld.cvut.cz 